\title{
Photoconductivity of an InAs/GaAs self-assembled quantum dot photoconductive $\mathrm{THz}$ antenna
}

\author{
Amit Yadav ${ }^{1}$, Andrei Gorodetsky ${ }^{1,2}$, Eugene Avrutin ${ }^{3}, K_{\text {Ksenia A. Fedorova }}{ }^{1}$, Edik U. Rafailov ${ }^{1}$ \\ 1. Optoelectronics and Biomedical Photonics Group, AIPT, Aston University, Birmingham, B4 7ET, UK \\ 2. ITMO University, St. Petersburg 197101, Russia \\ 3. Department of Electronics, University of York, York, YO10 5DD, UK
}

A broadband terahertz (THz) source is desirable for applications such as imaging, spectroscopy and security. Towards this, an InAs/GaAs quantum dot (QD) based photoconductive antenna (PCA) is a promising and compact solution for $\mathrm{THz}$ generation. Coherent $\mathrm{THz}$ radiation in the pulsed and the $\mathrm{CW}$ regime has been generated with a QD PCA under a resonant and off-resonant pumps [1,2]. While photoconductivity of QD materials in mid- and far-IR at lower temperatures has been studied for cryogenic sensors and attributed to interlevel transitions, nearIE interband photoconductivity needs further investigation $[3,4]$. In this work, we report on the photoconductive properties of an InAs/GaAs QD PCA pumped by a broadly-tunable InAs/GaAs QD external-cavity diode laser.

A coplanar stripline InAs/GaAs QD PCA with a 50- $\mu \mathrm{m}$ photoconductive gap and $240 \mathrm{~nm}$ thick contact pads was used for this study. The PCA active region structure contained 40 equally spaced InAs/GaAs QD layers grown on an undoped GaAs substrate by molecular beam epitaxy in the Stranski-Krastanow mode. The QD sizes were designed to have the ground state (GS), the first and the second exited states (ES1 and ES2) at $1218 \mathrm{~nm}, 1158 \mathrm{~nm}$, and $1141 \mathrm{~nm}$, respectively. The investigated PCA was pumped with a broadly-tunable $(1128 \mathrm{~nm}-1310 \mathrm{~nm})$ InAs/GaAs QD external-cavity diode laser (see Fig. 1a), similar to the reported in [5]. A cryostat (Janis CCS-450) was used to investigate the photoconductivity of the antenna at temperatures between $13 \mathrm{~K}$ and $400 \mathrm{~K}$, and a semiconductor characterisation system (Keithley 4200 SCS) was used to apply a voltage sweep and record a photocurrent.
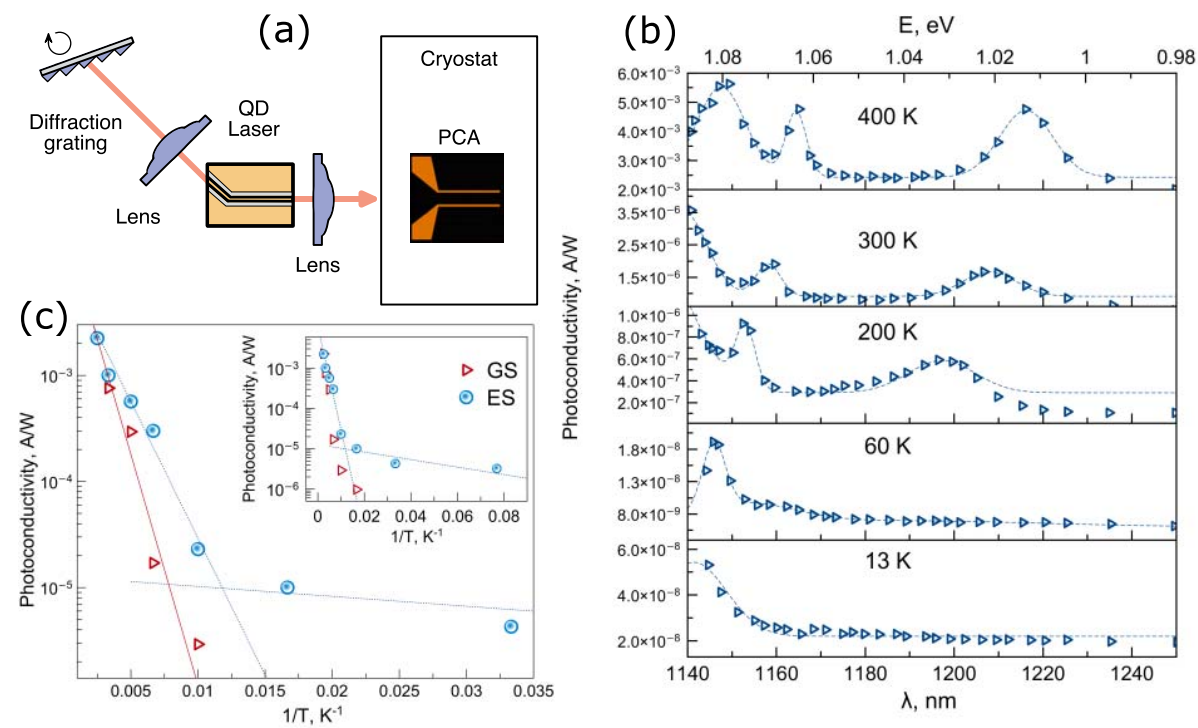

Fig. 1 (a) Schematic of the experimental setup, (b) QD PCA Photoconductivity at different temperatures, and (c) Inverse temperature dependence of GS and ES photoconductivity.

The temperature dependent photoconductive spectra (Fig. 1b) obtained by recording I-V curves of the QD PCA at various pump wavelength show distinct peaks for the GS, ES1, and, for higher temperatures, ES2. The inverse temperature dependence of the GS and ES1 conductivity is shown in Fig. 1c. The GS peak evolution is fitted with an activation dependence with an activation energy around $65 \mathrm{meV}$. However, this energy is greater than the energy separation between the GS and the ES1 and between the ES1 and the ES2, so thermal loss of carriers from the excited states is likely to affect photocurrent generation. The carrier escape from the excited level is activated at high temperatures, but it saturates at $\mathrm{T}<80 \mathrm{~K}$, which can be attributed to field assisted tunnelling being the main escape mechanism at low temperatures.

\section{References}

[1] R. R. Leyman, et al., "Quantum dot materials for terahertz generation applications," Laser Phton. Rev. 10(5), 772-779 (2016).

[2] T. Kruczek, et al., "Continuous wave terahertz radiation from an InAs/GaAs quantum-dot photomixer device," Appl. Phys. Lett. 101, 081114 (2012).

[3] S. Maimon, et al., "Intersublevel transitions in InAs/GaAs quantum dots infrared photodetectors," Appl. Phys. Lett. 73(14), 2003 (1998).

[4] J. Phillips, et al., "Far-infrared photoconductivity in self-organized InAs quantum dots," Appl. Phys. Lett. 72(16), 2020 (1998).

[5] K.A. Fedorova, et.al., "Broadly tunable high-power InAs/GaAs quantum-dot external cavity diode lasers," Opt. Exp. 18, 19438 (2010). 